\title{
LA MINERÍA DE TAJO A CIELO ABIERTO EN MÉXICO: UNA NUEVA FORMA DE COLONIALISMO
}

\author{
Luis Tamayo \\ CIDHEM, México
}

http://dx.doi.org/10.5209/rev_NOMA.2014.v44.n4.49291

\begin{abstract}
Resumen.- La minería de Tajo abierto se ha extendido como plaga por gran parte del territorio latinoamericano. En México, en particular, el caso es particularmente grave pues, según estimaciones, una cuarta parte de su territorio está concesionado a empresas mineras transnacionales. En este ensayo se estudia el caso de la minera Esperanza Silver, SA de CV, la cual pretendía establecerse en Temixco, Morelos y cuyo permiso de explotación fue recientemente rechazado por la Secretaría de Medio Ambiente y Recursos Naturales de la nación mexicana, no sin antes presentarse una importante oposición a la minera por parte de la sociedad civil organizada y el gobierno local.
\end{abstract}

Palabras clave.- Minería a tajo abierto, colonialismo, cambio climático.

\begin{abstract}
Surface mining has spread like a pest on big part of the Latin-American territory. In Mexico the case is particularly serious because the fourth part of his land is awarded to mining transnational companies. In this essay we studied the case of the corporation Esperanza Silver, SA de CV, which was trying to settle in Temixco, Morelos (Mexico) and whose permission of development was pushed back recently by the Ministry of Environment and Natural Resources (SEMARNAT) of the Mexican nation, not without an important opposition to the mine for the civil society and the local government.
\end{abstract}

Keywords.- Surface mining, colonialism, climate change.

\section{Introducción}

\author{
Nos pierde \\ la codicia de los menos \\ la cobardía de los más \\ la irracionalidad de todos \\ falta lenguaje \\ para decir \\ el horror que viene. (Riechmann, 2012)
}

En nuestros días, y causa de la inestable situación económica mundial derivada del fin de la era de los recursos ilimitados, ${ }^{1}$ el precio de los metales preciosos se ha incrementado de manera inusitada, ${ }^{2}$ lo cual ha abierto el camino a una de las industrias más depredadoras de los ecosistemas en toda la historia humana: la minería de tajo a cielo abierto.

\footnotetext{
1 Para poner un ejemplo proveniente de la propia minería recordemos lo enunciado por Javier Rodríguez Prado (2007): "En el año 1900, Estados Unidos extraía minerales de cobre con una ley promedio del $5 \%$, que en la actualidad es inferior al $0,4 \%$; diferencia abismal que indica la escasez del 'recurso'. Este ejemplo se repite en casi todas las geografías y tipos de minerales."

${ }^{2}$ Para la minería a tajo abierto es suficiente con que un yacimiento ofrezca 0.3 de gramo de oro por tonelada de mineral removido (y contaminado) para que la mina sea rentable. La mera existencia de la minería a tajo abierto moderna es una prueba clara del fin de la, denominada por W. Catton (2010, pag. 109), "era de la exuberancia".
} 
La minería de tajo a cielo abierto de metales preciosos es aquella que, para obtener el mineral a explotar, no excava en la búsqueda de la veta, sino que remueve con explosivos toda la superficie del terreno, baña con sustancias peligrosas el mineral (habitualmente con cianuro) para separar los componentes preciosos y construye lagunas de lixiviados que permanecen contaminadas durante siglos, las cuales, con el paso del tiempo y un inadecuado manejo, pueden llegar a contaminar los acuíferos de toda la región.

Esa forma de minería se ha extendido en todas aquellas naciones cuyas leyes o la corrupción de sus dirigentes les han permitido el acceso. De tal manera, por buena parte del mundo y en casi todo el territorio latinoamericano, se han establecido empresas de minería a tajo a cielo abierto que depredan los ecosistemas y empobrecen a las poblaciones indígenas (pues una vez concluidas las explotaciones mineras sólo dejan páramos yermos donde el cultivo otrora posible deviene un sueño). Empresas mineras que no solamente contaminan acuíferos y suelos sino que enferman a los pobladores de regiones enteras. Han sido muy pocas las naciones latinoamericanas (como Costa Rica ${ }^{3}$ o Bolivia ${ }^{4}$ ) las que, con enormes dificultades, han puesto límites al flagelo que representa la minería de tajo a cielo abierto de metales preciosos.

\section{La minería a tajo a cielo abierto: una prueba del fin de la era de la exuberancia}

La humanidad, por sus depredadores hábitos de consumo, ha generado en nuestros días una crisis ambiental sin precedentes, una denominada por el Prof. William Catton, el fundador de la Sociología ambiental en la Universidad de Chicago, el "fin de la era de la exuberancia". ${ }^{5}$ Y los responsables de tal crisis no son sólo las grandes empresas ecocidas o los tecnólogos que les hacen el juego, sino prácticamente todos los habitantes de la tierra, los cuales parecemos desconocer el principio de responsabilidad descrito hace medio siglo por Hans Jonas ${ }^{6}$ y gritado hasta el cansancio por Günther Anders. ${ }^{7}$

Las consecuencias de ese desconocimiento, con el paso de los años, se han revelado catastróficas: muy pocos de nuestros científicos y tecnólogos se preguntan acerca de los efectos que acarreará a la naturaleza producir tal o cual compuesto,

\footnotetext{
${ }^{3}$ El 4 de noviembre del 2010 el Congreso de Costa Rica prohibió, y de manera unánime, la minería a cielo abierto en su país.

${ }^{4}$ Gracias al presidente Evo Morales, la nación boliviana ha logrado escapar bastante de la minería a cielo abierto. Y lo hizo de una manera clara. Primero nacionalizó la mina de Sinchi Wayra (subsidiaria de la suiza Glencore), después la empresa canadiense South American Silver, para, al final, plantear una solución muy inteligente: no tuvo necesidad de establecer leyes que impidiesen la explotación de dichas minas (lo cual habría sido interpretado negativamente por los jerarcas de la economía globalizada y las propias mineras asentadas en Bolivia), solamente indicó que, como Bolivia era una nación soberana, tenía derecho a una participación también soberana de las ganancias derivadas de la riqueza del subsuelo boliviano, disposición que hizo, para las empresas transnacionales, inviable económicamente el establecimiento de la minería de tajo abierto de metales preciosos en suelo boliviano (aunque la minería del uranio sigue insistiendo).

${ }^{5} \mathrm{La}$ era de la exuberancia fue aquella en la cual los energéticos baratos permitieron la explosión demográfica y la aparición de la idea del progreso infinito, es decir, de que "siempre puede haber más para todos" (es decir, el "mito de los recursos ilimitados"), Catton, 2010, pags. 109ss.

6 "Obra de tal modo que los efectos de tu acción sean compatibles con la permanencia de una vida humana auténtica en la Tierra" (Jonas, 1995).

${ }^{7}$ Anders, 2002.
} 
establecer tal o cual procedimiento industrial o lo que producirá tal o cual artefacto; ${ }^{8}$ muy pocos ciudadanos se preguntan de dónde proviene la enorme cantidad de bienes que disfrutan, si su producción es "sustentable" o qué harán con las residuos sólidos que restan después de consumir los diversos artículos que el mercado globalizado ofrece.

Como consecuencia, al mismo tiempo que nos llenamos de comodidades, el mundo se cubre de una cantidad creciente de monstruos, del gas mostaza a la bomba atómica, del unicel a las dioxinas, ${ }^{9}$ de los pesticidas y herbicidas inorgánicos a los Organismos Genéticamente Modificados, de los contaminantes procedimientos para extraer petróleo a los aún peores de las grandes mineras de oro y plata modernas.

Nuestra naturaleza se ha convertido en un depósito de cantidades cada vez mayores de productos que los ecosistemas no pueden digerir, enfermando prácticamente a todas las especies de la tierra. ${ }^{10}$

Muchos de nuestros científicos y tecnólogos se volvieron cómplices de la destrucción del mundo orquestada por las grandes corporaciones ecocidas, esas que no se preocupan de la humanidad (pues no forman parte de ella) sino solamente por generar ganancias a sus inhumanos accionistas.

Las corporaciones florecen donde el neoliberalismo se impone, dominando no sólo el mercado, sino la vida de los hombres. El neoliberalismo hace a tales corporaciones hiperpotentes y, a pesar de ello, ciegas, pues carecen generalmente de visión de largo plazo. Ello es así pues la ganancia económica (su razón de ser) raramente es de largo plazo. $Y$ dividen al mundo en pobres (la enorme mayoría de los seres humanos) ${ }^{11}$ y ricos (los dueños y accionistas de las corporaciones), incrementando la desigualdad.

El medioambiente también se deteriora por el agotamiento acelerado de los productos de la tierra, de los energéticos a los minerales, de los bosques a las selvas y manglares, del agua limpia de los ríos y lagos a la belleza prístina de los glaciares y arrecifes de coral.

$Y$, en tal proceso, hasta los nombres se modifican: la madre tierra se convierte en "sustrato"; la explotación desmedida en "producción", las catástrofes derivadas de nuestra depredación son llamadas "naturales" y las especies hermanas que no son aprovechables por la industria (sean animales o vegetales) son consideradas cosas "inútiles" y por ende soslayables.

Muchos de los científicos y tecnólogos que colaboraron con las principales corporaciones del mundo consideran que nada tuvieron que ver con la destrucción del planeta cuando desde el comienzo carecieron del principio de responsabilidad por cometer el enorme error de participar en corporaciones que consideraban al otro

\footnotetext{
${ }^{8}$ With nearly 80,000 chemicals on the market in the United States, many of which are used by millions of Americans in their daily lives and are un- or understudied and largely unregulated, exposure to potential environmental carcinogens is widespread. Only a few hundred of the more than 80,000 chemicals in use in the United States have been tested for safety". "Many known or suspected carcinogens are completely unregulated" (Leffall, L. y Kripke, M., 2010, pag. ii).

${ }^{9}$ Nombre como se conoce habitualmente la tetraclorodibenzodioxina.

${ }^{10}$ Cfr. el Informe del President's Cancer Panel (Lefall, L. y Kripke, M., 2010).

11 El 99\% de la humanidad, según decían los "indignados" apostados en Wall Street.
} 
y al mundo como ajenos. Y el resto de los ciudadanos nos hacemos cómplices de tal error al consumir los degradados productos que tales corporaciones ofertan.

Como consecuencia de todo ello, la humanidad es colocada ante una amenaza de dimensiones nunca antes vista: la catástrofe ambiental derivada del calentamiento global y el agotamiento de los recursos naturales (el fin de la era de la exuberancia), en cual los metales tienen un papel central. La moderna minería a tajo a cielo abierto de metales preciosos es una consecuencia directa del agotamiento de las "vetas" minerales, pues el incremento de los precios de los metales preciosos derivado de su agotamiento ha conducido, con el objeto de cubrir las necesidades del mercado, a que florezcan empresas que, mediante el procedimiento antes citado, ofrecen los hipervaluados metales. ${ }^{12}$

\section{Las corporaciones mineras: represión, corrupción y depredación}

El negocio de esa minería tóxica es, además de una respuesta desesperada ante el fin de los recursos de la tierra, un negocio muy productivo. En la nación mexicana incluso el narcotráfico ha ingresado a ese negocio. ${ }^{13}$ En el estudio "La Red de Control Corporativo Global" (The Network of Global Corporate Control), tres investigadores de la Universidad de Zürich, Stefania Vitali, James B. Glattfelder y Stefano Battiston (2011) indicaron, después de estudiar más de 40 mil corporaciones transnacionales, que:

Un pequeño grupo de 147 grandes corporaciones, principalmente financieras y minero-extractivas, en la práctica controlan la economía global.

Dicha afirmación fue ampliada en el estudio "El 1\% global: desenmascarando a la clase dominante transnacional" (The Global 1\%: Exposing the Transnational Ruling Class, Censored Notebook, 22 de agosto de 2012), donde dos integrantes de la Sonoma University, Peter Phillips y Kimberly Soeiro, cuidadosamente investigaron a la minera Freeport-McMoran, la cual explota depósitos enormes de minerales en Indonesia, Sudamérica y África. Sólo en 2010, indica el estudio, dicha empresa vendió 3.9 mil millones de libras de cobre, 1.9 millones de onzas de oro y 67 millones de libras de molibdeno, todo lo cual le reportó ingresos por casi 19 mil millones de dólares y una renta neta de más de 4 mil millones de USDlls. La empresa, sólo en Papua, Indonesia, emplea a 23,000 trabajadores con salarios por debajo de los 3 dólares la hora. Cuando en septiembre de 2011 dichos trabajadores se fueron a la huelga exigiendo mejorías en salarios y condiciones de trabajo (pues se enteraron de que el estándar internacional de los salarios para los mineros era entre 17 y 43 dólares por hora y no los 3 que ellos recibían), la policía pagada por la minera los reprimió violentamente. El conflicto escaló y las fuerzas armadas mataron a varios huelguistas. Y la lealtad de las fuerzas armadas para con la minera estaba basada en la corrupción: en noviembre del 2011, Camelia Pasandaran (2011) del diario Jakarta Globe, denunció que las fuerzas de seguridad indonesias de Papua recibían dinero de la minera (10 millones de dólares anuales, aproximadamente). La cuestión ha sido tan grave que la Comisión de Seguridad e

\footnotetext{
${ }^{12}$ Me permito denominar a tales metales preciosos cono "hipervaluados" pues la enorme mayoría de ellos no van a parar a la industria sino a las arcas de los bancos y a la joyería. Según indica Rodríguez Pardo, el 98\% del oro extraído por la minería moderna es para acumularse en las arcas de los bancos $(80 \%)$ o para producir artículos suntuarios (18\%): "Un anillo de oro produce 18 toneladas de escombros" (Rodríguez Pardo, 2009).

${ }^{13}$ Mayorga, 2013.
} 
Intercambio de los USA está revisando la responsabilidad de la minera bajo la Ley sobre Prácticas Extranjeras Corruptas de Estados Unidos. Y los muertos por los enfrentamientos sumaron varias decenas, mientras que los heridos y encarcelados lo eran por centenares. Y mientras todo ello ocurría, la minera seguía operando con otros trabajadores, protegida por las fuerzas armadas indonesias. $Y$ hechos similares han ocurrido en Perú, Argentina, Guatemala y muchas otras naciones. En buena parte del mundo las empresas mineras que extraen metales vía el tajo abierto contaminan, dañan al tejido social y, una vez implantadas no pueden ser expulsadas pues su enorme capital compra fácilmente las voluntades:

El 8 de agosto de 2011, Karishma Vaswani, de la BBC de Londres, reportó que la corporación minera de EEUU Freeport-McMoran ha sido acusada de todo, desde contaminar el ambiente al financiamiento de la represión en sus cuatro décadas de operaciones en la provincia indonesia de Papua... Pregúntele en la calle a cualquier papuano lo que piensa de la minera Freeport y le dirá que la corporación es una ladrona, dijo Nelels Tebay, un pastor de Papua y coordinador de la red de la paz de Papua (Phillips y Soeiro, 2012).

Por otro lado, en varias naciones sudamericanas la explotación de metales preciosos a tajo abierto es una experiencia cotidiana. De Yanacocha, la mayor explotación de oro a cielo abierto del mundo, en Perú, hasta la Patagonia (en Pascua Lama, en la frontera entre Chile y Argentina), empresas transnacionales se apropian de los metales y dejan la depredación atrás. No son respetados ni los derechos de los pueblos ni la salvaguarda de la naturaleza (los glaciares de Pascua Lama).

En su estudio Vienen por el oro, vienen por todo, Javier Rodríguez Pardo (2009) estudia con cuidado los terribles efectos que las mineras Meridian Gold y Barrick Gold (ambas provenientes de Canadá) producen en Argentina. Y su investigación no deja de denunciar a los funcionarios argentinos coludidos con el gran capital, así como a las naciones que se dicen preocupadas por el cuidado del medioambiente cuando en realidad apoyan la depredación del mundo:

Barrick llegó a la Argentina a principios de los años noventa para lanzar el proyecto Pascua Lama, un proyecto binacional compartido con Chile, pero antes que eso, la más monstruosa explotación de oro y cobre del mundo, a cinco mil metros de altura y sobre la superficie de por lo menos tres glaciares, el Toro 1, Toro 2 y Esperanza, todos del lado chileno. "Esto es lo que desemboca en la ley de glaciares y el posterior veto. El glaciar Guanaco, que es donde Barrick dice que trasladará con palas mecánicas el hielo de Toro 1, Toro 2 y Esperanza, lo comparten la Argentina y Chile [...] A principios de mayo de 2009, la Barrick Gold puso en marcha Pascua Lama, primer emprendimiento minero binacional del mundo, compartido entre la Argentina y Chile. Con una inversión de USDlls 3,000 millones, la empresa estima que producirá entre 750 mil y 800 mil onzas de oro y 35 millones de onzas de plata por año a un costo de caja de entre USDlls 200/250 por onza. La construcción del proyecto, que tendrá una vida útil de 25 años, terminará a fines de 2012. La producción comenzará en 2013. Cuando funcione en pleno, utilizará un millón de litros de agua por hora en una zona semidesértica, detonará 45 mil toneladas de roca por día y tendrá un dique de cola (basurero 
químico) de 420 hectáreas y 200 metros de profundidad. Es, sin duda, el proyecto más polémico de las centenas de emprendimientos mineros que afloraron en el país como consecuencia de los enormes beneficios legales e impositivos que ofrece la Argentina a las multinacionales de la industria minera. "Será un desastre, peor que cinco plantas de celulosa juntas" (Rodríguez Pardo, 2009).

Y si en Argentina y Perú la situación es dramática, el caso de la minería tóxica de metales preciosos es en México particularmente grave: gracias a la apertura económica que representó el Tratado de Libre Comercio de América del Norte (TLCAN) firmado por el ex presidente Carlos Salinas de Gortari con sus pares de USA y Canadá, ${ }^{14}$ durante las administraciones de los ex presidentes Vicente Fox (2000-2006) y Felipe Calderón (2006-2012) fueron concesionadas a la industria minera más de 50 millones de hectáreas del territorio mexicano, y otorgados más de 25 mil permisos para la exploración, explotación y producción de metales preciosos y otros minerales. $^{15}$

Y, como en Indonesia, en México es también la corrupción la que ha abierto el camino a dichos proyectos mineros pues la extracción de cualquier metal precioso o mineral cuesta a las compañías mineras apenas entre 5 y 111 pesos anuales por hectárea. $^{16}$ El Estado mexicano no recibe prácticamente nada al concesionar regiones enteras (al menos no abiertamente) ni exige a las mismas la restauración exitosa de los sitios impactados 0 , siquiera, cumplir con las leyes vigentes. ${ }^{17}$ Tales empresas trasnacionales encuentran en México una sencilla fuente de enriquecimiento a costa del envenenamiento de ecosistemas y pobladores. ${ }^{18}$ Tales corporaciones no hacen sino confirmar las tesis que Carl Amery (2002) presenta en su estudio Auschwitz, comienza el Siglo XXI? Hitler como precursor: Hitler sigue vivo en la ideología de las modernas corporaciones transnacionales, las cuales son capaces de destruir al mundo todo si beneficia a sus intereses económicos. ${ }^{19}$

Y aquí no sobra recordar las palabras del ex Presidente chileno Salvador Allende ante la Organización de las Naciones Unidas del 4 de diciembre de 1972, el cual, para muchos, constituyó la razón fundamental de su asesinato:

Estamos ante un verdadero conflicto frontal entre las grandes corporaciones y los Estados. Éstos aparecen interferidos en sus decisiones fundamentales políticas, económicas y militares- por organizaciones globales que no dependen de ningún Estado y que en la suma de sus actividades no responden ni están fiscalizadas por ningún Parlamento, por ninguna institución representativa del interés colectivo. En una palabra, es toda la estructura política del mundo la que está siendo socavada. Pero las grandes empresas transnacionales no sólo atentan contra los intereses genuinos de los países en

\footnotetext{
${ }^{14}$ Siguiendo, por supuesto, las indicaciones del Banco Mundial y las del Banco Interamericano de Desarrollo (Rodríguez Pardo, 2009).

${ }^{15}$ Cfr. Mijangos Leal (2013).

${ }^{16}$ Y 111 pesos mexicanos son, en la cotización actual, jmenos de 10 USDlls!

${ }^{17} \mathrm{De}$ hecho, las empresas mineras, dado su enorme poderío económico se permiten, una vez asentadas en una comunidad, desobedecer abiertamente las leyes mexicanas como ha demostrado la Minera San Xavier en San Luis Potosí, México, la cual desobedeció en varias ocasiones a las autoridades locales cobijada por políticos corruptos, Cfr. Juan Carlos Ruiz Guadalajara (2012).

${ }^{18}$ Cfr. Hersch, 2013b.

${ }^{19}$ Cfr. también: Balkan, 2004.
} 
desarrollo, sino que su acción avasalladora e incontrolada se da también en los países industrializados donde se asientan. Ello ha sido denunciado en los últimos tiempos en Europa y Estados Unidos, lo que ha originado una investigación en el propio Senado norteamericano. Ante este peligro, los pueblos desarrollados no están más seguros que los subdesarrollados. Es un fenómeno que ya ha provocado la creciente movilización de los trabajadores organizados, incluyendo a las grandes entidades sindicales que existen en el mundo. Una vez más, la actuación solidaria internacional de los trabajadores, deberá enfrentarse a un adversario común: el imperialismo (Allende, 1972).

\section{La minera Esperanza Silver}

Tal y como está asentado en sus documentos, la minera Esperanza Silver de México, filial de Esperanza Resources, es una corporación que "está dedicada a la exploración y desarrollo de propiedades mineras en México", e incluye las concesiones de El sabinal en Chihuahua (24,000 Has) y la del Cerro jumil, Esperanza (15,000 Has) en Temixco, Morelos. La Esperanza Resources Corp., además, posee y explota minas en Perú y Chile.

La empresa Esperanza Silver S.A. de C.V., cuyo capital es principalmente canadiense $^{20}$ y cuenta con el apoyo del gobierno de esa nación, ${ }^{21}$ decidió, a inicios del Siglo XXI, explorar la región central del Estado de Morelos para averiguar si era posible establecer una explotación de oro y plata en la región (Temixco, Miacatlán, Alpuyeca, Cuentepec). Dicha concesión incluía, de manera asombrosa, en su quinta etapa, al Sitio Arqueológico de Xochicalco, el cual fue declarado por la UNESCO, en el año 1999, Patrimonio cultural de la humanidad. La concesión minera de la Esperanza Silver es de 15,000 hectáreas a explotarse en 7 etapas y se ubica en una región con múltiples asentamientos humanos. ${ }^{22}$ Dichos asentamientos, buena parte de los cuales presenta grados de marginación alta y economía de subsistencia, se localizan dentro de un radio menor a los $4 \mathrm{~km}$, y su límite norte se encuentra a escasos $12 \mathrm{~km}$ de la ciudad de Cuernavaca. Cabe señalar además, que la cabecera de la pista del Aeropuerto Internacional Mariano Matamoros, se encuentra a escasos metros del límite nororiental de la concesión.

\section{El proyecto de la Esperanza Silver en Temixco, Morelos}

La primera etapa del proyecto de explotación de la mina pretendía realizarse los cerros El Jumil y Colotepec, en un área de 696.92 hectáreas pertenecientes a la

\footnotetext{
${ }^{20}$ Cfr. Esperanza Resources Corp. (2012).

${ }^{21} \mathrm{Al}$ respecto refiere la editorial del número 21 de En el volcan (Revista de los trabajadores del Instituto Nacional de Antropología e Historia de Morelos): "En 2012, Stephen Harper, en el marco de la Cumbre de las Américas (Cartagena de Indias, Colombia), afirmó: "vemos la industria minera canadiense creciendo en las Américas, algo que será beneficioso para la prosperidad mutua y será prioridad de nuestro gobierno...En el futuro próximo vemos una mayor inversión canadiense en recursos naturales en las Américas; esto es algo que será bueno para nuestra prosperidad y es una prioridad de nuestro gobierno." Fuera de la retórica, tanto al gobierno de Harper, como a las mineras, lo que realmente les interesa es el crecimiento acelerado y seguro de su tasa de ganancia que producirán sus corporaciones".

${ }^{22}$ La Unidad Habitacional José Ma. Morelos y Pavón, los pueblos de Cuentepec, Xochitepec, Alpuyeca, Xochicalco y El Rodeo, todos ellos pertenecientes a los municipios de Temixco, Xochitepec y Miacatlán.
} 
comunidad de Tetlama, Morelos y a medio kilómetro de la zona arqueológica de Xochicalco antes referida. ${ }^{23}$

La Manifestación de Impacto Ambiental (MIA) presentada por la empresa minera a fines del 2012 a la Secretaría de Medio Ambiente y Recursos Naturales (SEMARNAT) indicaba que la explotación se realizaría bajo la técnica de tajo abierto, por lo que pretendía "remover" (en términos latos: destruir), tan sólo en la primera etapa, casi 500 hectáreas de selva baja caducifolia, un ecosistema extraordinario que en dicha región contiene diversas especies de flora y fauna endémicas y en peligro de extinción. $Y$ todo ello ante los ojos azorados de buena parte de la comunidad científica y ambientalista del Estado de Morelos, la cual se esforzaba en comprender los límites planetarios ${ }^{24}$ y en encontrar las mejores y más eficientes medidas para mitigar el Calentamiento Global Antropogénico, ${ }^{25}$ una de las cuales es, precisamente, la preservación de los mismos bosques y selvas que la minera pretendía "remover".

\section{La región a explotar: el centro de Morelos $^{26}$}

El Estado de Morelos es una región muy particular de la nación mexicana. Su capital, Cuernavaca, fue denominada a principios del Siglo XIX por Alexander von Humboldt, "la ciudad de la eterna primavera" por su espléndido clima templado y las maravillosas barrancas que la atraviesan y actúan como sistemas de refrigeración naturales (producto del encuentro de dos ecozonas: la neártica y la neotropical). Esa ventaja climática, aunada a su cercanía con la Ciudad de México, la convirtieron en sitio de elección para innumerables centros de investigación científica mexicanos. La Ciudad de Cuernavaca, han señalado varios estudios, tiene una cantidad de científicos e investigadores por metro cuadrado similar a la de varias ciudades europeas. $^{27}$

Y esa masa crítica no podía dejar de reflejarse en la participación ciudadana y, por la belleza natural de la región, en una muy particular: el cuidado del medioambiente.

Desde la época mesoamericana, la región noroeste de Morelos (Cuauhnáhuac) era muy valorada por su clima y belleza escénica. Diversos pueblos mesoamericanos se establecieron en la región (Tlahuicas, Mexicas) y sabían de la importancia del cuidado del Gran Bosque de Agua. ${ }^{28}$ Dicho bosque, que se encuentra al suroeste de la capital de México y parte, al norte, de las ciénegas del Río Lerma e incluye los bosques del sur la misma capital, del poniente del Estado de México y el norponiente

\footnotetext{
${ }^{23}$ Cfr. Hersch (2013a).

${ }^{24}$ Rockström, 2009; Riechmann, 2012b.

${ }^{25}$ Sarmiento, 2013.

${ }^{26}$ Una parte de este apartado fue presentada en la el ensayo "El impacto de la minería de tajo abierto en la recarga de los acuíferos de Morelos y la Ciudad de México", Conferencia DAAD Alumni México 2013: Retos para el manejo adecuado de los recursos naturales en una ciudad de millones de habitantes, Ciudad de México, 14 de junio de 2013.

${ }^{27}$ En su estudio Morelos: capital del conocimiento, el Dr. Medardo Tapia (del CRIM/UNAM) indica: "En 2004 el CONACyT reconocía que el Estado de Morelos era la segunda entidad del país con más investigadores nacionales, sólo después del Distrito Federal. Proporcionalmente el Estado de Morelos tiene cinco veces más investigadores nacionales por habitante que Jalisco y Nuevo León, así como nueve veces más que el Estado de México (Tapia, 2006, pag. 72).

${ }^{28} \mathrm{El}$ Gran Bosque de Agua parte de las Ciénegas del Lerma, en el estado de México y llega hasta la Sierra del Ajusco-Chichinautzin en Morelos y el Distrito Federal, J. Hoth (2012); Marin, L. El agua en México: retos y oportunidades (http://www.aguaenmexico.org/images/aguareto.pdf).
} 
de Morelos, es donde se forma el acuífero de la región lacustre donde se hallaba Tenochtitlan, la cual se recargaba de agua dulce desde el sur, de los lagos de Xochimilco ${ }^{29}$ y Chalco, ${ }^{30}$ así como el principal acuífero de Morelos: el acuífero de Cuernavaca, uno de los cuatro existentes en el Estado.

Durante la Colonia española, el Estado de Morelos explotó su riqueza en acuíferos mediante el establecimiento de Haciendas azucareras, las cuales fueron muy exitosas durante varios siglos. Entretanto, la capital del Estado, Cuernavaca, se llenaba de las quintas de los más prósperos integrantes de la sociedad mexicana e internacional, así como de espacios recreativos y de investigación. Hasta el 2005, la Ciudad de Cuernavaca contaba con 39 Centros de investigacion nacionales y era el segundo destino mundial para el aprendizaje de la lengua castellana... y ello solamente por su clima y paisaje. ${ }^{31}$

Y el cuidado del medioambiente también se reflejaba en las instituciones políticas: Morelos fue uno de los primeros Estados de la nación mexicana en contar con una Secretaría de medioambiente. ${ }^{32}$ En Morelos numerosos pueblos originarios y organizaciones ambientalistas luchan por la preservación de sus manatiales, de los sitios históricos, bosques y barrancas, ${ }^{33}$ luchas que fueron particularmente complejas (e incluso violentamente reprimidas ${ }^{34}$ ) durante los 12 años en los cuales el gobierno neoliberal del Partido Acción Nacional (PAN) gobernó la entidad. ${ }^{35}$

Afortunadamente, a partir del segundo semestre del 2012 ocurre un cambio de gobierno ${ }^{36}$ en Morelos que permite que un importante grupo de ambientalistas se involucre en la nueva administración, todo lo cual conduce a cambios muy importantes en las políticas estatales en materia de medioambiente. Como

\footnotetext{
${ }^{29}$ De ahí que no podemos estar más de acuerdo con el Dr. Luis Zambrano: ¡es urgente el rescate de Xochimilco! (Cfr. http://www.dgcs.unam.mx/boletin/bdboletin/2012_798.html).

${ }^{30}$ Ezcurra, 1990, pag. 12.

${ }^{31}$ Lo cual se ha, desgraciadamente, lastimado en la actualidad debido a la guerra establecida por el ex Presidente Felipe Calderón en contra del narco (2006-2012), lo cual ha convertido a la otrora tranquila Cuernavaca, en una de las ciudades más peligrosas del país.

${ }^{32}$ La "Secretaría de Desarrollo Ambiental", fundada por la Dra. Úrsula Oswald Spring en 1998. Esa Secretaría fue degradada, en el 2000, por el gobierno panista de Sergio Estrada Cajigal a "Comisión Estatal de Agua y Medio Ambiente (CEAMA)" para que pudiese ser dirigida por Javier Bolaños, el primer Director de dicho organismo. Ello ocurrió asi pues tal gobernador exigía que el encargado del medioambiente fuese Bolaños aunque su amigo no contase con el nivel profesional requerido para fungir como Secretario de Desarrollo Ambiental Estatal (de facto estaba muy lejos de poseer la preparación de la Dra. Úrsula Oswald). Durante su gestión, Bolaños permitió el ingreso del gran capital inmobiliario (Casas Geo, Ara, Tecnourbe) y cubrió buena parte del Estado de Morelos de casitas de "interés social" (actualmente deshabitadas en su mayoría), las cuales implicaron negocios millonarios a los gobiernos panistas de Vicente Fox, Felipe Calderón y sus aliados morelenses.

${ }^{33}$ Un ejemplo de ello fue documentado por el cineasta Francesco Taboada en el filme: 13 pueblos en defensa del agua, el aire y la tierra, México, 2008.

${ }^{34} \mathrm{Vgr}$. la lucha, a inicio del siglo XXI, contra la entrega al gran capital del "Casino de la Selva", ese emblemático ex Hotel y casino que menciona Malcolm Lowry en su novela Bajo el volcán y que fue destruido para establecer jun bodegón Price Cosco!

${ }^{35}$ Como reacción contra los 70 años en los cuales el Partido Revolucionario Institucional (PRI) gobernó el país. Desgraciadamente, los gobiernos panistas no tardaron demasiado en mostrar que ¡podían ser peores que el PRI! Ello se reflejó claramente en el castigo ciudadano que sufrió el pAN en las elecciones del 2012.

${ }^{36}$ En julio del 2012 fue elegido como Gobernador de Morelos el Lic. Graco Ramírez Garrido Abreu (del Partido de la Revolución Democrática, de centro-izquierda), el cual colocó, como su Secretario de Desarrollo Sustentable al Mtro. Topiltzin Contreras Macbeath, un reconocido científico y ambientalista morelense.
} 
consecuencia de ello, tan pronto se conoce la noticia de que la empresa minera Esperanza Silver había solicitado a la federación el permiso de explotación en su concesión morelense, la sociedad civil organizada, la academia y el gobierno del Estado de Morelos muy rápida, y eficazmente, se opusieron a dicho proyecto.

\section{La lucha contra la minera}

Como señalamos anteriormente, la empresa minera venía solicitando permisos de exploración a la delegación de la Secretaría de Medioambiente y Recursos naturales (SEMARNAT) local desde hacía una década, pagando a los comuneros de la región por los permisos y comprando voluntades de caciques y autoridades locales.

Cuando el nuevo gobierno estatal (a través de la Secretaría de Desarrollo Sustentable) y los ambientalistas de Morelos se enteran —a fines del 2012 - de la cercana aprobación de la solicitud de explotación de la minera, la Esperanza Silver llevaba una fuerte ventaja: había comprado la voluntad de buena parte de los caciques y la comunidad de Tetlama (el sitio donde se desarrollaría la primera etapa de la explotación) por lo que, una vez aprobada su solicitud por la Dirección General de Impacto y Riesgo Ambiental (DGIRA/SEMARNAT), el cambio de uso de suelo (la siguiente etapa del trámite) sería relativamente sencillo (por tener de su lado a los tetlamitas y porque el gobierno anterior nunca quiso publicar el Programa de Ordenamiento Ecológico y Territorial de la región pues atentaba contra los intereses de esa y otras empresas trasnacionales).

En consecuencia, la sociedad civil organizada, apoyada por la Secretaría de Desarrollo Sustentable estatal y múltiples instancias ciudadanas y académicas, ${ }^{37}$ establece el Movimiento Morelense Contra la Minería de Metales Preciosos (MMCMMP) ${ }^{38}$ el cual realizó caravanas, marchas, visitas a funcionarios federales e internacionales (como a la embajada de Canadá) entre muchas otras tareas. Los festivales de cine ${ }^{39}$ y los mass media locales también cumplieron un importante papel en la denuncia contra la minera, en particular el Diario de Morelos y La Jornada Morelos. ${ }^{40} \mathrm{Y}$ todo ello sin demeritar las declaraciones públicas que continuamente realizaron tanto el Gobernador estatal como su Secretario de Desarrollo Sustentable, así como el Rector de la Universidad Autónoma del Estado de Morelos (UAEM), ${ }^{41}$ los cuales criticaron abiertamente al proyecto minero.

\footnotetext{
${ }^{37}$ Como la Red Mexicana de Justicia Socioambiental, el Consejo Consultivo para el Desarrollo Sustentable (núcleo Morelos) de la SEMARNAT, la Universidad Autónoma del Estado de Morelos, investigadores del INAH Morelos, la Academia de Ciencias de Morelos y la Academia de Ciencias Sociales y Humanidades del Estado de Morelos.

${ }^{38}$ El MMCMMP incluye, además de los investigadores referidos en la nota 1 de este ensayo, a activistas de la talla de Flora Guerrero (presidente de los Guardianes de los árboles), Saúl Roque y Juan Jaramillo (del Consejo de Pueblos de Morelos), Beatriz Lemus Kourchenko y Anabel Ferreira, entre muchos otros.

${ }^{39}$ Como el Cinema Planeta 2013 dirigido por Eleonora Izunza de Pech y Gustavo Martínez Ballesté, los cuales cerraron dicho Festival con el film: Corazón de cielo, corazón de tierra (Black/Sandig, México/Guatemala, 2011), un bellísimo documento fílmico donde se denuncia la destrucción que la minería a cielo abierto realiza en Guatemala.

40 Y en los cuales el apoyo de las periodistas Lya Gutiérrez Quintanilla y Gina Batista fue fundamental.

${ }^{41}$ El Dr. Alejandro Vera Jiménez.
} 


\section{Las razones de los opositores}

En síntesis, los opositores a la solicitud de la Esperanza Silver arguyeron los siguientes elementos contra el proyecto de explotación minera: ${ }^{42}$

1. Afectará y contaminará los recursos hídricos de Cuernavaca y otros municipios morelenses. El flujo hídrico, tanto superficial como subterráneo, sería uno de los recursos más severamente impactados, y esto no sólo por la enorme cantidad de agua que las minas de tajo abierto requieren para la lixiviación del mineral (que realizan con cianuro), ${ }^{43}$ sino también porque una vez terminada la explotación (lo cual ocurrirá en poco más de una década) los remanentes minerales pueden contaminar severamente el agua y los suelos de toda la región. El proyecto minero se encuentra muy cerca del río Los Sabinos (algunos tramos del mismo quedan dentro de la poligonal del proyecto), por lo que la contaminación que generaría la mina tendría repercusiones en la biodiversidad y los recursos hídricos de toda la región. Dicha afirmación se fundamenta en que el sitio destinado a la lixiviación se encuentra dentro de la "Formación Cuernavaca", unidad hidrogeológica que forma parte del acuífero del mismo nombre y cuyas fuentes de aprovechamiento (manantiales y pozos) sostienen a una parte importante de la población de la Zona Metropolitana de Cuernavaca, la Ciudad de México y extensas zonas de riego agrícola. Dentro del Estado de Morelos, esta región recibe importantes precipitaciones que alimentan el acuífero mencionado, y sus escurrimientos se integran a las subcuencas de los ríos Apatlaco y Tembembe, tributarios del Río Amacuzac, todos ellos sujetos al Programa de Rescate del Río Apatlaco, emprendido por autoridades del gobierno federal, estatal y municipal. Por lo tanto, contaminar sus aguas con cianuro y liberar en ellas metales pesados afectará la salud de una gran cantidad de pobladores. Cabe señalar que, según el informe técnico 2011 de la Comisión Nacional del Agua (CONAGUA), el acuífero de Cuernavaca está en vías de sobreexplotación, lo cual ratificó recientemente el Director de la Comisión de Agua de Morelos, el Dr. Juan Carlos Valencia (Excélsior, 7 de abril del 2013).

2. Afectará la salud de los habitantes y los ecosistemas de toda la región. El proyecto minero, dado que se realizará bajo la tecnología de tajo abierto, no sólo dañará la salud de los habitantes al contaminar sus acuíferos sino que, como utiliza explosivos para fracturar el mineral antes de bañarlo con cianuro, en cada voladura en el área del tajo produciría una columna de polvo y compuestos del material explosivo, la cual, tal y como ha ocurrido en otros lugares de nuestro país y del mundo, dañará gravemente la salud de los

\footnotetext{
${ }^{42}$ En lo que sigue refiero los principales elementos de la declaratoria emitida luego del Foro sobre la minería tóxica realizado en la UAEM el 29 de mayo del 2013 y organizado por la Dra. Laura Ortiz, la Dra. Lilian González y el que estas líneas suscribe.

${ }^{43}$ Según indicó el Ing. Antonio García Ramón del Colegio de Ingenieros Civiles, sección Morelos, se deriva de la Manifestación de impacto ambiental entregada por la empresa minera a la SEMARNAT que el "Consumo aproximado de agua según sus datos: 12 lt/m2 hr X 591,600m2 = 7,099,200 de litros por hora. Si suponemos que el Consumo de agua por persona por día es de 150 litros resulta que: 7,099,200 / $150=47,328 \mathrm{dias} / \mathrm{hr}$. Años que se alimentaria una persona 47,328 / 8,760=5.4 años por cada hora de consumo en la mina esto sin considerar otros consumos de agua en la mina". Es decir, concluye el estudio del Ing. García Ramón: "en 10 horas de trabajo de la mina se consume el equivalente a 54 años de la vida de una persona" (Foro sobre la minería tóxica, realizado en el Auditorio Emiliano Zapata de la Universidad Autónoma del Estado de Morelos, 29.05.2013).
} 
habitantes y ecosistemas de la región. Tales polvaredas afectarán sustancialmente, asimismo, al Aeropuerto Internacional Mariano Matamoros, el cual se encuentra en los límites de la poligonal concesionada. Respecto al manejo de sustancias peligrosas, es necesario mencionar que las minas de tajo abierto siempre ponen en riesgo a las poblaciones aledañas por los escapes de cianuro al medio ambiente, los cuales ocurren durante las operaciones de extracción.

3. Dañará al tejido social. La empresa Esperanza Silver tiene comprada con migajas y promesas a buena parte de la población de Tetlama (en el municipio de Temixco) y eso ha conducido a enfrentamientos con pobladores de las regiones fuera de la poligonal de la primera etapa del proyecto, pero cuyas tierras también serán afectadas en el futuro por la explotación minera (vgr. Miacatlán, Alpuyeca o Cuernavaca). La empresa minera es capaz de mentir abiertamente al pueblo tetlamita y a las autoridades estatales y federales mexicanas (vgr. uno de los representantes de la minera, el Lic. Hugo Rosales Gavilla, se atrevió a decir en la consulta pública del 21 de febrero en la ex Hacienda de Temixco, que la explotación minera "no dañaría al medioambiente" cuando, apenas unos minutos antes, otro de sus colaboradores había sostenido que en las casi 700 has de la primera etapa despojarían totalmente de su cubierta vegetal a casi 500 has. Una cubierta vegetal que, no sobra decirlo, está compuesta por plantas perennes que a la naturaleza llevó siglos desarrollar).

4. Constituye un acto de colonialismo. La minería de tajo a cielo abierto de metales preciosos no es sino un expolio a la nación mexicana realizado por empresas transnacionales que se han coludido con políticos corruptos (pues la nación mexicana y las comunidades afectadas, como antes indicamos, no obtienen sino apenas migajas de los enormes beneficios que tales empresas recogen). Tal proyecto minero se realizaría, además, en un contexto legal muy desfavorable para la ciudadanía. La Ley minera vigente en México, asimismo, parece no entender que la minería moderna, al menos la que opera bajo la tecnología de tajo a cielo abierto, no permite concesiones como las ahora otorgadas, pues afecta los intereses y daña la salud de los pobladores de las regiones donde se asienta. La minería de tajo abierto ya no es subterránea sino superficial y, por ende, afecta no sólo a ecosistemas y acuíferos sino a los pobladores y a sus parcelas. La minería de tajo a cielo abierto, por tanto, no es compatible con otros usos del suelo (como la agricultura, la habitación o la recreación).

5. Afectará al Sitio arqueológico de Xochicalco. El proyecto minero, además, causaría un grave impacto al entorno natural y visual de la zona arqueológica de Xochicalco al ser devastados los cerros aledaños. Asimismo, en el norte de Xochicalco hay grandes cavernas cuyos techos están formados por capas de rocas muy fracturadas y por consiguiente, vulnerables a las vibraciones causadas por las explosiones que generaría la mina, lo que destruiría una parte importante de la ciudad de Xochicalco, incluida la cueva del Observatorio, ubicada al Norte de la Acrópolis.

6. La minera Esperanza Silver, al término de su operación (después de poco más de una década) sólo dejará la peor pobreza y destrucción en la región 
afectada pues, al retirar la cubierta vegetal centenaria de cientos (e incluso miles si tomamos en cuenta las 7 etapas del proyecto) de hectáreas, construirá un enorme desierto en pleno centro del Estado de Morelos. La Empresa Esperanza Silver ocasionará la desertificación de una amplia región del Estado de Morelos pues la operación de las empresas mineras no garantizan, de ninguna manera la reforestación correcta en las regiones que devastarán. Dicha empresa sostiene, de manera totalmente irresponsable, que es capaz de restaurar la Selva baja caducifolia de su concesión "en tan sólo dos años", mostrando que no tienen la más mínima idea de lo que implica restaurar ese ecosistema (lo cual no logran ni los mejores centros de investigación forestal del mundo). La Selva baja caducifolia es un ecosistema muy peculiar. Está conformado por plantas perennes, las cuales en el estiaje se secan en su parte superficial manteniendo sin embargo la vitalidad de las dos terceras partes de sus raíces. Eso produce que cuando se visita la Selva baja caducifolia en el estiaje se tiene la impresión de que la cubierta vegetal de la misma está muerta en casi su totalidad... pero apenas empieza la temporada de lluvias, la vegetación se recupera instantáneamente, lo cual es una consecuencia de que una tercera parte de las raíces de las plantas siguieron vivas durante el estiaje. No es sino una estrategia adaptativa el que tales plantas se sequen superficialmente durante la temporada seca. Eso les permite soportar el elevado asoleamiento y, a la vez, mantener la vitalidad de una tercera parte de sus raíces. Es gracias a esa estrategia adaptativa que la selva baja caducifolia se renueva año con año y nos regala varios meses de verdor. Sin sus sabias y profundas raíces perennes, tales plantas no podrían, de ninguna manera, sobrevivir. La empresa minera, desconociendo lo anteriormente referido, pretende, primero, retirar casi la totalidad de la cubierta vegetal superficial de sus "concesiones", luego, explotar, pulverizar y lavar con cianuro y agua el mineral para, acto seguido, depositarlo en verdaderas montañas de "Jales" (Ios cuales contaminarán el suelo durante siglos) y, finalmente, cubrir con algunos centímetros de tierra y sus correspondientes plantitas (la pretendida "reforestación") la superficie devastada. Las empresa minera parece desconocer (o quizás si lo sabe pero lo oculta) que el elevado asoleamiento de la región no permitirá el crecimiento de tales plantas. Asimismo que, cuando lleguen las lluvias (en su mayoría torrenciales a causa del calentamiento global), la cubierta de tierra que coloquen será arrastrada por ellas, generando una erosión tal que producirá que la región pierda sus suelos y cubierta vegetal, es decir, la empresa minera lo único que haría es crear un enorme desierto en pleno centro del Estado de Morelos.

\section{Conclusiones}

Dado que el proyecto de la empresa Esperanza Silver (y ahora de la Álamos Gold, pues adquirió la concesión cuando la DGIRA/SEMARNAT rechazó la MIA de la Esperanza Silver) además de desertificar y expoliar la región, afectaría la cantidad y calidad de los acuíferos que nutren a la Ciudad de Cuernavaca y muchos otros pueblos de Morelos, buena parte de los habitantes de dichos asentamientos 
humanos no pudieron sino oponerse a proyecto tan ecocida, ${ }^{44}$ a esa nueva forma de colonialismo que tal empresa "canadiense" 45 pretendía.

En consecuencia, el viernes 7 de junio del 2013, el Secretario de Desarrollo Sustentable del Estado de Morelos, Topiltzin Contreras MacBeath, informó que el C. Alfonso Ramírez Flores, titular de la Dirección General de Impacto y Riesgo Ambiental de la SEMARNAT, como una consecuencia de la presión ejercida por la ciudadanía y el gobierno de Morelos, así como por la calidad de las razones opuestas al proyecto de la Esperanza Silver, negó a la empresa el permiso de explotación minera, lo cual constituye una muy importante victoria de la ciudadanía morelense sobre el gran capital ecodepredador. ${ }^{46}$

\section{Epílogo}

Alguna vez dijo Bertrand Russell: no entiendo porqué la humanidad comete siempre los mismos errores... jcon la cantidad de errores nuevos que podríamos cometer! (Galeano, 2011).

La cuestión carece, desgraciadamente como siempre, de un final plenamente feliz: a mediados de junio del año en curso, mientras que por un lado se realizaba, en Cuernavaca, la preaudiencia "Despojo y envenenamiento de las comunidades por minería y basura" del Tribunal Permanente de los Pueblos, capítulo México, ${ }^{47}$ al mismo tiempo se reunían, frente a las oficinas del gobierno de Morelos, un importante grupo de telamitas, los cuales reclamaban fuese revisado nuevamente el proyecto minero de la Esperanza Silver. Y tales telamitas no dejaron de amenazar al gobierno estatal con el cierre de carreteras y del Sitio arqueológico de Xochicalco (como si el gobierno de Morelos hubiese emitido el dictamen y no la DGIRA, una instancia federal cuya tarea es vigilar que los proyectos a implantarse en México no dañen a los habitantes). $Y$ son esos mismos telamitas los que amenazaron y difamaron (aduciendo que actuaban por interés económico) a los activistas antiminera $^{48}$ y ahora festejan que la empresa Álamos Gold, una minera más grande que la anterior, recupere el proyecto y pretenda realizarlo de nuevo.

Y mientras esos telamitas exigían sea abierta "su" mina para así obtener "sus" empleos, otros mexicanos, los que ya han sufrido los impactos de dichas empresas mineras, se reunían ante el Tribunal Permanente de los Pueblos para quejarse por la contaminación de sus acuíferos, por la destrucción de su tierra, por el envenenamiento de sus hijos.

\footnotetext{
${ }^{44}$ Tamayo, 2010.

${ }^{45}$ Me permito poner entre comillas el vocablo "canadiense", el cual se ha utilizado en numerosos documentos periodísticos, pues como bien indica Karl Marx, y este caso no es la excepción, el gran capital carece de nacionalidad. Muchos integrantes del pueblo canadiense también contemplan azorados la destrucción que las corporaciones transnacionales realizan, al cobijo de su nombre, en todo el planeta.

${ }^{46}$ Esta victoria no es la primera que la sociedad mexicana obtiene sobre una minera. El 21 de mayo del 2012, el pueblo veracruzano logró que la DGIRA de la SEMARNAT negase el cambio de uso de suelo al proyecto "Caballo blanco" de la minera Goldgroup Inc. En tal caso la victoria fue sencilla pues dicha mina pretendía establecerse (y realizar sus constantes y enormes explosiones) ja apenas 3 Kms de la Planta nuclear de Laguna verde!

${ }^{47}$ Organizado en México por Andrés Barreda, Raúl García Barrios y Fernanda Campa, entre otros.

${ }^{48}$ Algunos investigadores del Instituto Nacional de Antropología e Historia han debido responsabilizar públicamente a la empresa minera por cualquier daño que pudiesen sufrir en sus personas o bienes (lo cual, desgraciadamente, ya ha empezado a ocurrir).
} 
En el México contradictorio de nuestros días se manifiestan las fuerzas más opuestas. Los tetlamitas comprados por la empresa minera exigen se apruebe el proyecto de la Esperanza Silver y ello a sabiendas del daño que causaría a su salud. Nunca olvidaré la ocasión en la que, cuando la Consulta pública realizada el 21 febrero de 2013 sobre el proyecto minero, el Dr. Horacio Riojas ${ }^{49}$ habló a los tetlamitas de las enfermedades que contraerían a causa de la minera, a lo que varios de éstos respondieron: "No nos importa, de algo nos tenemos que morir".

Y tienen razón, de algo tenemos que morir todos... aunque personalmente preferiría que nuestros hijos no enfermasen o muriesen a causa de procesos o sustancias emitidas por empresas que solo depredan la tierra y, además, engañan a las comunidades indígenas solamente para enriquecerse... y no sólo a ellas timan, pues como bien señala Jorge Riechmann (2012a: 171):

Como en otras dimensiones de la crisis socioecológica, en lo referente al calentamiento climático se nos escapa la rapidez de los cambios movidos por dinámicas de crecimiento exponencial: nuestra intuición no está a la altura. No nos damos cuenta de lo que está pasando... y además hay poderosos grupos de interés que hacen cuanto pueden para que sigamos sin darnos cuenta.

Para finalizar no puedo dejar de referir que, hacia finales de diciembre del 2014, la empresa Esperanza Silver Corp. inició un juicio de amparo contra el Programa de Ordenamiento Ecológico y Territorial del Estado de Morelos, ${ }^{50}$ el cual recogió la exigencia ciudadana antes expresada e impide la realización de proyectos mineros de Tajo a cielo abierto de metales preciosos en suelo morelense. En consecuencia, desde el Secretario de Desarrollo Sustentable hasta el que estas líneas suscribe, nos encontramos actualmente demandados legalmente por la empresa minera y teniendo que defender los intereses del pueblo de México. El proceso está en curso.

\section{Referencias bibliográficas:}

Allende, S. (1972). Discurso ante la Organización de las Naciones Unidas. http://www.abacq.net/imagineria/cronolo4.htm

Amery C. (2002). Auschwitz, comienza el Siglo XXI? Hitler como precursor. Madrid: Turner/Fondo de Cultura Económica.

Anders, G. (2002). L'obsolescence de l'homme. Paris: Encyclopedie des nuisances/lvrea.

Balkan, J. (2004). The Corporation: A Pathological Pursuit of Profit and Power.New York: Free Press/Simon and Schuster.

Catton, W. (2010). Rebasados. México: Océano.

Esperanza Silver de México (2012). Manifestación de Impacto Ambiental, Modalidad Particular, Mina Esperanza, Tetlama, Morelos. México: Planeación y Proyectos de Ingeniería,

S.C.,

Ordóñez

Profesional.

http://app1.semarnat.gob.mx/dgiraDocs/documentos/mor/estudios/2012/17MO2012 M0005.pdf

\footnotetext{
${ }^{49}$ Investigador del Instituto Nacional de Salud Pública (INSP) de México.

${ }^{50}$ Publicado en el Diario oficial del Estado de Morelos Tierra y libertad el 29 de septiembre del 2014.
} 
Esperanza Resources Corp. (2012). Consolidated Financial Statements. For the years ended December 31, 2012 and 2011. http://www.epzresources.com/_resources/pdfs/Annual_Report_2012.pdf

Ezcurra, E. (1990). De las chinampas a la megalópolis. México: FCE.

Galeano, E. (2011, 27 de julio). Megaminería, saqueo e indignación. Entrevista a Eduardo Galeano. http://www.youtube.com/watch?v=NmFTGnHE0OY Hersch, P. (2013a). "Agujeramos o agujeramos: los caminos para conseguir lo que se quiere. El caso de la Minera La Esperanza Silver en el cerro El Jumil, municipio de Temixco, Morelos". En el volcán 18. http://www.enelvolcan.com/feb2013/225agujeramos-o-agujeramos-los-caminos-para-conseguir-lo-que-se-quiere-el-caso-dela-minera-la-esperanza-silver-en-el-cerro-el-jumil-municipio-de-temixco-morelos Hersch, P. (2013b). "Efectos patológicos previsibles de la iniciativa de minería a tajo abierto en el cerro El Jumil, Temixco, en la salud pública". En el Volcán 19. http://www.enelvolcan.com/mzo2013/236-efectos-patologicos-previsibles-de-lainiciativa-de-mineria-a-tajo-abierto-en-el-cerro-el-jumil-temixco-en-la-salud-publica Hoth, J. (Ed) (2012). ECOBA 2012, Estrategia regional para la conservación del Bosque de Agua. México: Fundación Gonzalo Río Arronte/FBdelA/Pronatura. Jonas, H. (1995). El principio de responsabilidad: ensayo de una ética para la civilización tecnológica. Madrid: Herder.

Lasalle, L. y Kripke, M. (President's Cancer Panel) (2010). Reducing environmental cancer risk (2008-2009 Annual report). USA: U. S. Department of Health and Human Services/National Cancer Institute.

Mayorga, P. (2013, 25 de septiembre). "Madera, entre el despojo de mineras y el embate del narco". Revista Proceso, 1997. http://www.proceso.com.mx/?p=353692 Mijangos Leal, M.-A. (2013). "La nueva fiebre del oro. Comunidades afectadas por la empresa minera Goldcorp. Tribunal Popular Internacional de la Salud". En el volcan 21. http://www.enelvolcan.com/may2013/251-la-nueva-fiebre-del-oro-comunidadesafectadas-por-la-empresa-minera-goldcorp-tribunal-popular-internacional-de-la-salud Morales, E. (2010, 20 de abril). Discurso en Cochabamba, Bolivia. http://boliviacontaminada.blogspot.mx/2010/04/discurso-de-evo-morales-ayma-enla.html

Pasandaran, C. (2011, 7 de noviembre). "Striking Freeport Employees Lower Wage Increase Demands". Jakarta Globe, p. 1. http://www.thejakartaglobe.com/business/striking-freeport-employees-lower-wageincrease-demands $/ 476800$

Phillips, P., Soeiro, K. (2012). "The Global 1\%: Exposing the Transnational Ruling Class". Censored Notebook. http://www.projectcensored.org/top-stories/articles/theglobal-1-exposing-the-transnational-ruling-class/

Riechmann, J. (2012a). El socialismo puede llegar sólo en bicicleta. Madrid:

Catarata.

Riechmann, J. (2012b). Poemas lisiados (2009-2011). Madrid: La oveja roja. Rockström, J. (2009). "Planetary Boundaries: Exploring the Safe Operating Space for Humanity". Ecology and society 14 (2): 32.

http://www.ecologyandsociety.org/vol14/iss2/art32/

Rodríguez Pardo, J. (2007, 24 de julio). ¿Existe una minería posible?. Rebelion. http://www.rebelion.org/noticia.php?id=53959

Rodríguez Pardo, J. (2009). Vienen por el oro, vienen por todo. Bs. As: CICCUS. Ruiz Guadalajara, J-.C. (2012, 22 de octubre). "¿Quién se acuerda de Minera san Xavier?". La jornada. http://www.jornada.unam.mx/2012/09/22/opinion/018a1pol 
Sarmiento, A. (2013). “¿Visión "nueva” o visión correcta y a largo plazo?: Huexca y los riesgos ocultos de las plantas termoeléctricas". En el volcan 20.

http://www.enelvolcan.com/abr2013/241-ivision-nueva-o-vision-correcta-y-a-largoplazo-huexca-y-los-riesgos-ocultos-de-las-plantas-termoelectricas

Sklair, L. (2001). The Transnational Capitalist Class. Oxford: Blackwell.

Tamayo, L. (2010). La locura ecocida. México: Fontamara.

Tapia, M. (2006). Morelos: capital del conocimiento. México: CRIM/UNAM.

Vaswani, K. (2011, 9 de agosto). "US Firm Freeport Struggles to Escape Its Past in Papua”. Jakarta: BBC News. http://www.bbc.co.uk/news/world-asia-pacific-14417718

Vitali,S., Glattfelder, JB., Battiston, S. (2011, 26 de octubre). The Network of Global Corporate Control. Plos One.

http://www.plosone.org/article/info\%3Adoi\%2F10.1371\%2Fjournal.pone.0025995 